\title{
Development of a Green \& Sustainable Manufacturing Process for Gefapixant Citrate (MK-7264) Part 6: Development of an Improved Commercial Salt Formation Process.
}

Kevin M. Maloney, ${ }^{*}$ Si-Wei Zhang, * Anne E. Mohan, Alfred Y. Lee, Patrick Larpent, Hong Ren, Guy R. Humphrey, Richard Desmond, Michael DiBenedetto, Wenjun Liu, Ivan H. Lee, Eric Sirota, Michael J. Di Maso, Embarek Alwedi, Siqing Song, Hsieh Yao D. Chang

${ }^{1}$ Department of Process Research and Development, Merck \& Co., Inc., Rahway, New Jersey, 07065, United States

kevin maloney@merck.com

siwei.zhang@merck.com

\section{Table of Contents}

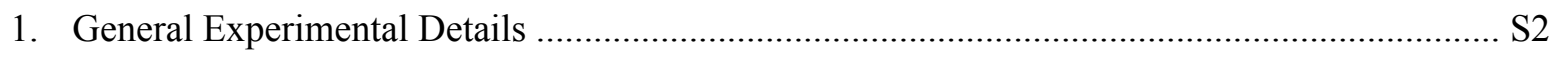

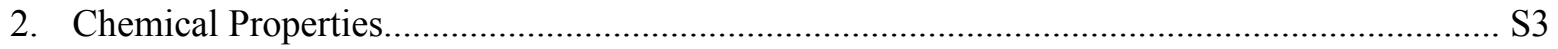

2.1. Dissociation constant

2.2. NMR Spectra

2.3. High Resolution Mass Spectrum 


\section{General Experimental Details}

Materials. Reagents were purchased in reagent grade from commercial suppliers and used without further purification, unless otherwise described. NMR solvent, specifically, $d_{6}$-DMSO (anhydrous, 99.9\% $d$ content, catalog 570672-50G), was purchased from Sigma-Aldrich and Cambridge Isotope Laboratories and used as received.

Solvent-Mediated Slurry Experiments. For the preparation of a suspension for solubility measurements or phase conversion, an excess amount of each solid was added to the selected solvent. The suspension was then held in a pie block and magnetically stirred at $750 \mathrm{rpm}$, on a Heidolph round hot plate stirrer (Schwabach, Germany). Aliquots of the suspension were withdrawn and filtered through a $0.22 \mu \mathrm{m}$ PTFE syringe filter. The phase of the solid residue was immediately determined after filtration by PXRD.

\section{Instrumentation.}

PXRD measurements were carried out on a Panalytical (Natick, MA, USA) X-pert Pro PW3040 diffractometer with an $\mathrm{X}^{\prime}$ celerator detector $(\mathrm{Cu} \mathrm{K \alpha}$ radiation, $45 \mathrm{kV}$ voltage, and $40 \mathrm{~mA}$ current). Approximately $5 \mathrm{mg}$ of powder was sprinkled on the surface of a zero background silicon (510) sample holder and scanned from 2 to $40^{\circ} 2 \theta$ at a speed of $8.4^{\circ} / \mathrm{min}$ and a step size of $0.02^{\circ}$.

DSC was conducted with a TA Instruments (New Castle, DE, USA) Discovery unit. About $3 \mathrm{mg}$ of sample in a pin-holed Tzero aluminum pan was heated at $10^{\circ} \mathrm{C} / \mathrm{min}$ under $50 \mathrm{~mL} / \mathrm{min} \mathrm{N} 2$ purge. TGA was conducted with a TA Instruments Q500 unit. About $8 \mathrm{mg}$ of sample in a Pt Pan was heated at $10{ }^{\circ} \mathrm{C} / \mathrm{min}$ under $55 \mathrm{~mL} / \mathrm{min} \mathrm{N} 2$ purge.

Proton nuclear magnetic resonance $\left({ }^{1} \mathrm{H}\right.$ NMR) spectra and carbon nuclear magnetic resonance $\left({ }^{13} \mathrm{C}\right.$ NMR) spectra were recorded at $25{ }^{\circ} \mathrm{C}$ (unless stated otherwise) on a Bruker 500 spectrometer using a liquid nitrogen-cooled triple resonance Prodigy ${ }^{\mathrm{TM}}$ CryoProbe. Chemical shifts for protons are reported in parts per million downfield from tetramethylsilane and are referenced to residual proton of the NMR solvent according to values reported in the literature. Chemical shifts for carbon are reported in parts per million downfield from tetramethylsilane and are referenced to the carbon resonances of the NMR solvent. For samples in $d_{6}$-DMSO the residual solvent signal was referenced to $2.50 \mathrm{ppm}$ for ${ }^{1} \mathrm{H}$ and $39.52 \mathrm{ppm}$ for ${ }^{13} \mathrm{C}$. Data are represented as follows: chemical shift, integration, multiplicity $(\mathrm{br}=\mathrm{broad}, \mathrm{s}=$ singlet, $\mathrm{d}=\mathrm{doublet}$, $\mathrm{t}=$ triplet, $\mathrm{q}=$ quartet, quint $=$ quintet, hept $=$ heptet, $\mathrm{m}=$ multiplet, om $=$ overlapping multiplets, $\mathrm{dd}=$ doublet of doublets, $\mathrm{ddd}=$ doublet of doublet of doublets, $\mathrm{dt}=$ doublet of triplets), coupling constants $(J)$ in Hertz (Hz). HRMS data were obtained using a Waters Acquity UPLC interfaced with a Waters Xevo G2 QTof ESI with the source temperature set to $120^{\circ} \mathrm{C}$.

Symbols. $\delta=$ parts per million $(\mathrm{ppm}), \mathrm{RMSD}=$ root mean square deviation. 


\section{Chemical Properties}

\subsection{Dissociation constant}

The dissociation constants determined for Gefapixant in ionic strength adjusted (ISA) water $(0.15 \mathrm{M} \mathrm{KCl})$ at $25^{\circ} \mathrm{C}$ are 6.4 for the pyrimidinium group and 10.2 for the sulfonamide group.

\subsection{NMR Spectra}
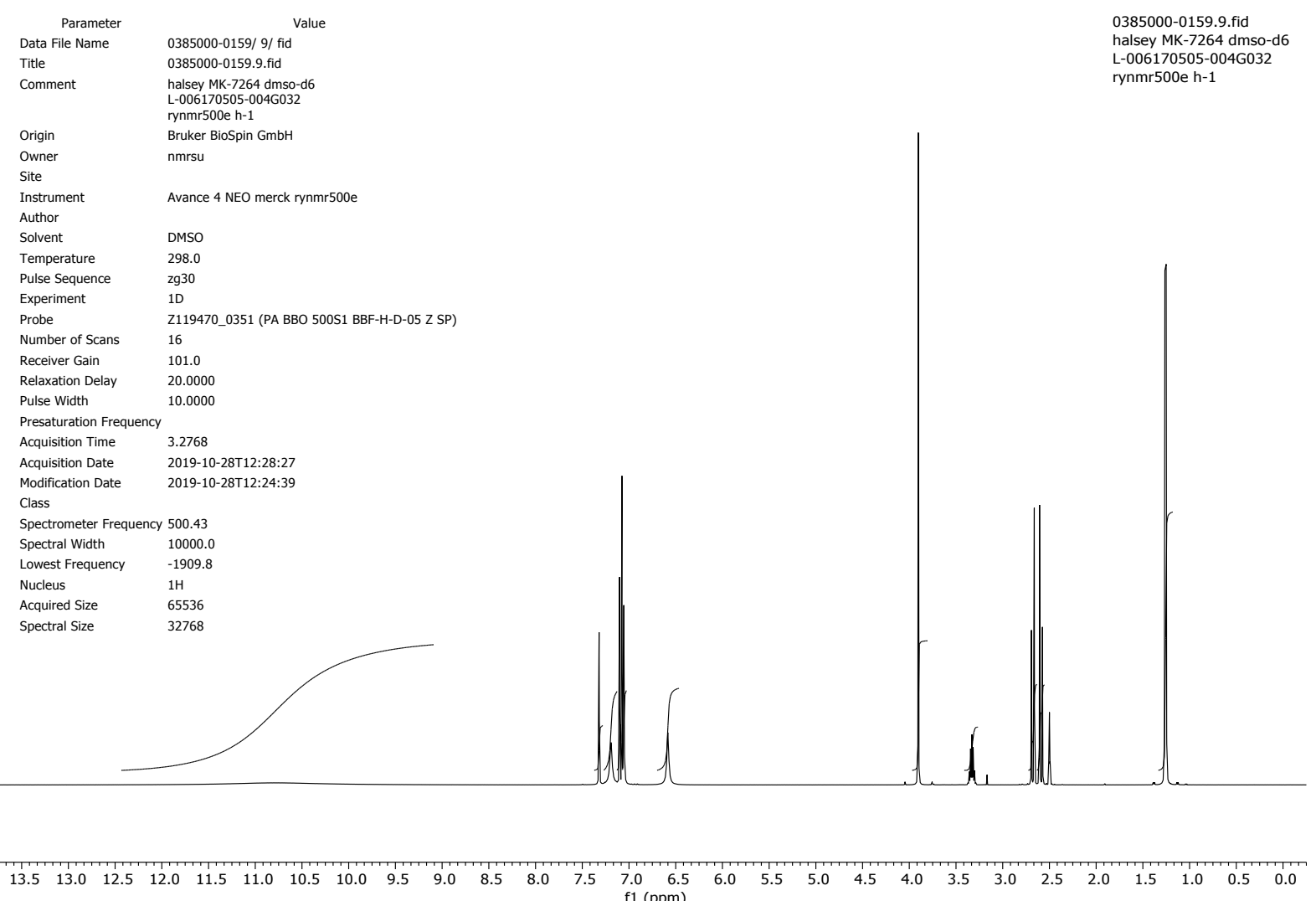

Figure S1. ${ }^{1} \mathrm{H}$ NMR $(500 \mathrm{MHz})$ spectrum of 1 in $d_{6}$-DMSO. 


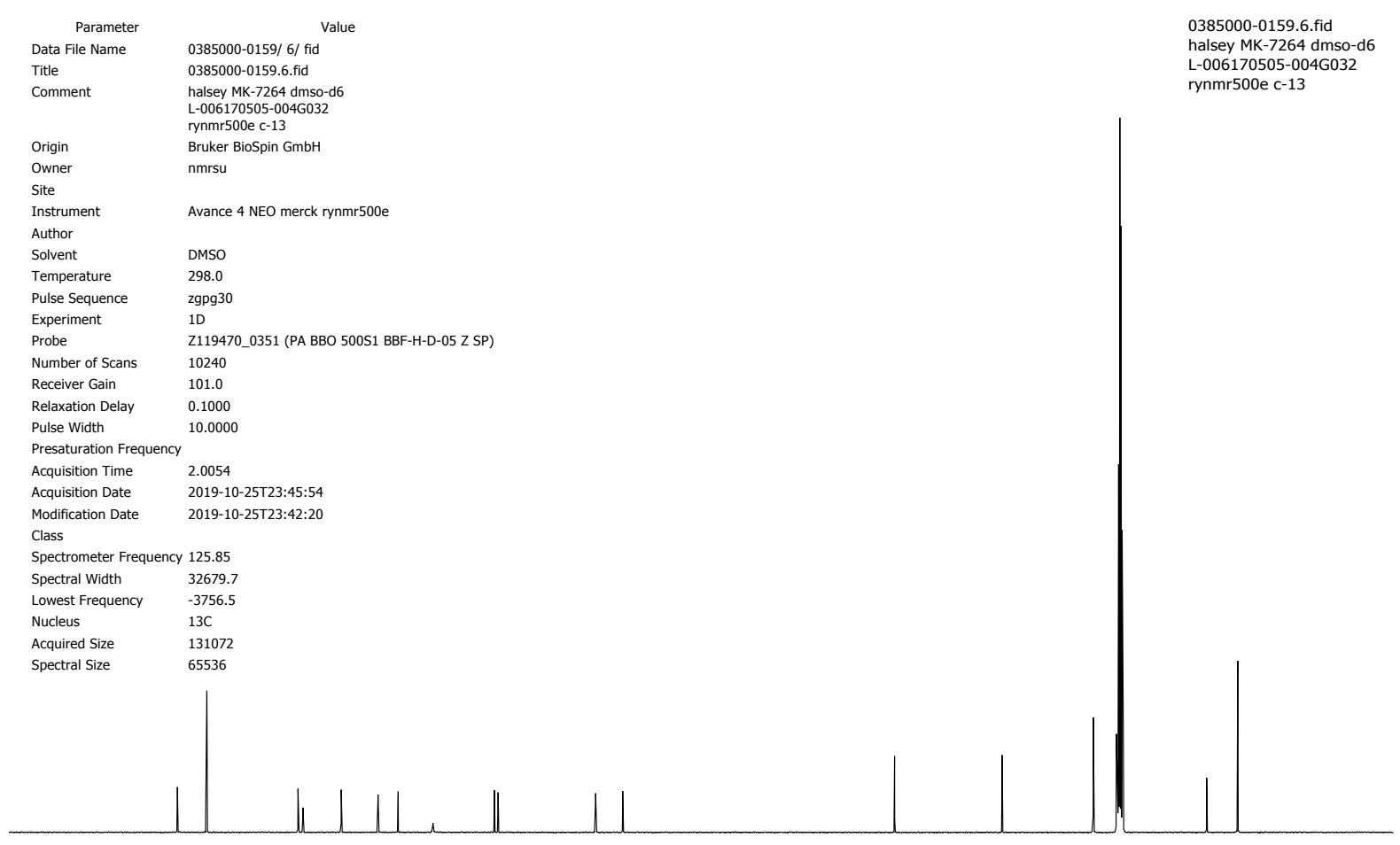

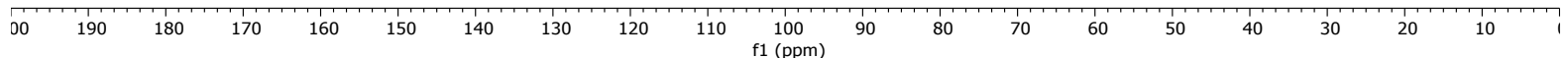

Figure S2. ${ }^{13} \mathrm{C}$ NMR $(126 \mathrm{MHz})$ spectrum of 1 in $d_{6}$-DMSO. 


\subsection{High Resolution Mass Spectrum}

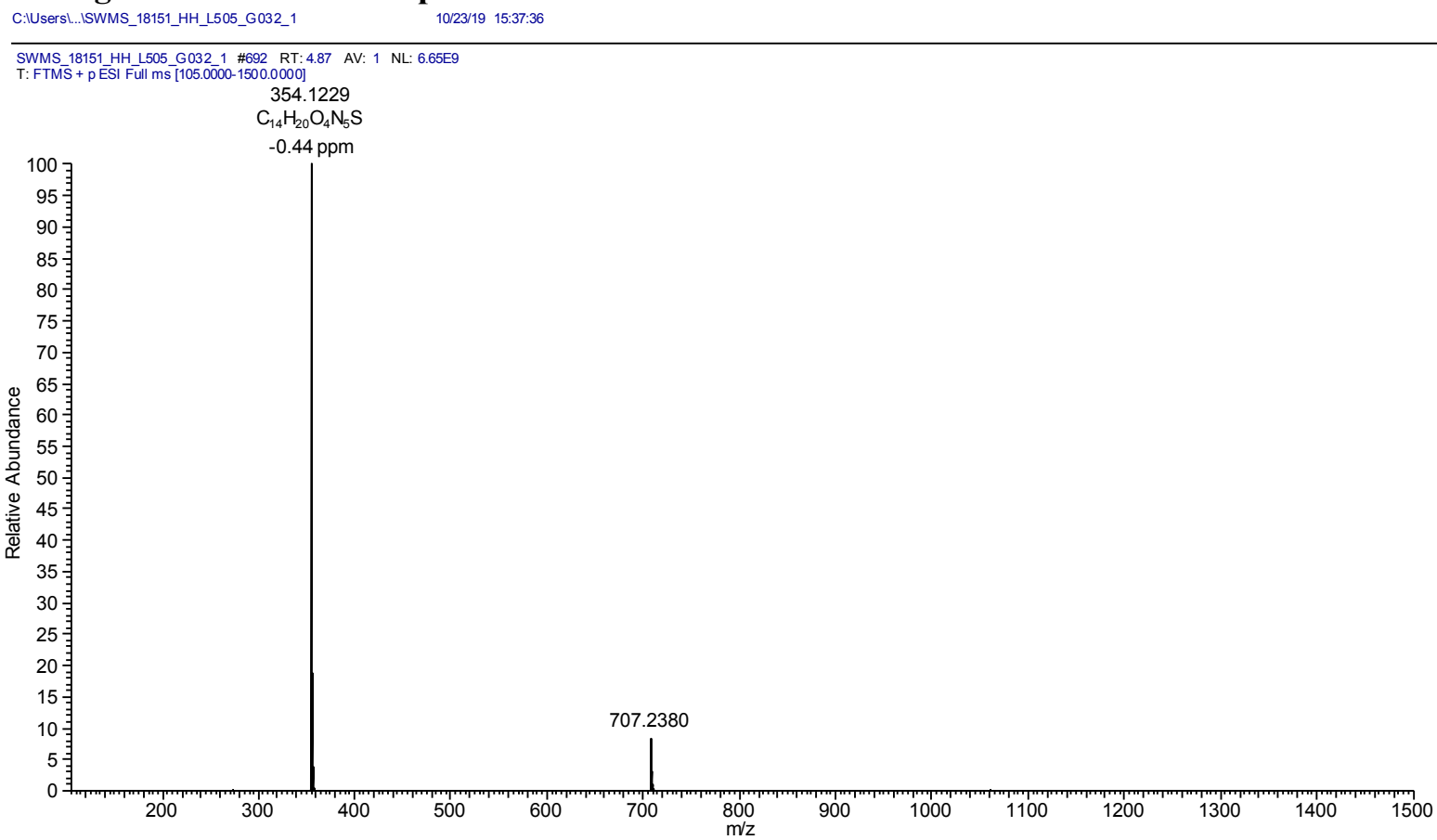

Figure S3. High Resolution Mass Spectrum. 